ÉGYPTE

monde arabe

\section{Égypte/Monde arabe}

19 | 2019

Les zabbâlîn, un objet surétudié ?

\title{
Introduction : les zabbâlîn, un objet sur-étudié ?
}

Introduction to the special issue: the Zabbaleen's community, an over-studied object?

\section{Pierre Desvaux}

\section{OpenEdition}

\section{Journals}

Édition électronique

URL : https://journals.openedition.org/ema/4452

DOI : $10.4000 /$ ema.4452

ISSN : 2090-7273

Éditeur

CEDEJ - Centre d'études et de documentation économiques juridiques et sociales

Édition imprimée

Date de publication : 1 janvier 2019

Pagination : 9-32

ISBN : 9782900956052

ISSN : 1110-5097

\section{Référence électronique}

Pierre Desvaux, «Introduction : les zabbâlîn, un objet sur-étudié ? », Égypte/Monde arabe [En ligne], 19 | 2019, mis en ligne le 01 janvier 2019, consulté le 07 juillet 2022. URL : http://journals.openedition.org/ ema/4452 ; DOl : https://doi.org/10.4000/ema.4452 


\section{Pierre Desvaux}

\section{INTRODUCTION : \\ LES ZABBÂLÎN, UN OBJET SUR-ÉTUDIÉ?}

«Une maison typique Navajo est un habitat indien où vivent huit anthropologues » (Sukarieh et Tannock, 2013)

\section{RÉSUMÉ}

Cette introduction propose un cadre analytique de la surétude appliquée à l'exemple des chiffonniers cairotes (connus sous le nom de zabbâlîn). Je cherche notamment à mettre en avant les conditions d'émergence des processus de surétude telles qu'étudiées par la littérature scientifique produite à ce sujet. Cette dernière fait ressortir des points concordants entre différents exemples de communautés touchées (marginalisation, expérience d'une forme de crise plus ou moins récente, localisation accessible) qui permettent de mieux comprendre les mécanismes de la médiatisation des zabbâlîn. Je propose également d'interroger les liens entre cette médiatisation importante de la communauté liée au travail de nombreux acteurs (chercheurs, journalistes, documentaristes, association de développement etc.) au regard de(s) économie(s) de production du savoir au sens large ainsi qu'aux biais entrepreneurialistes et orientalistes qui sous-tendent cet intérêt démesuré. 
L'objectif de ce numéro consiste à interroger les ressorts de l'importance démesurée de la représentation d'une communauté représentant environ 100000 personnes (Florin 2015) au sein d'une ville d'environ 20 millions d'habitants (Barthel 2017). Les chiffonniers du Caire, ou zabbâlîn en arabe, occupent une place particulière dans les études de science sociale consacrées à la capitale égyptienne, notamment le plus grand quartier où ils habitent et travaillent, Manshiyyat Nâsir (voir I'article de G. du Roy dans ce numéro). De plus, et au-delà de la fascination exercée par ce groupe social sur les chercheurs, on peut noter que l'attrait pour la communauté concerne également des journalistes (puisque d'innombrables articles de presse ont été écrits à leur sujet), des documentaristes ou encore des associatifs (religieux ou développementalistes) avec la présence importante et répétée dans le temps de multiples ONG et la mise en œuvre de projets de développement dans les zarraye $b^{1}$. Il s'agit ainsi de porter, à travers les contributions présentées ici, la réflexion sur les raisons ou les déraisons de cette fascination. S'il est difficile de quantifier une telle surreprésentation d'une communauté dans la constitution d'un champ de savoir et de visibilité impliquant plusieurs acteurs aux rationalités différentes (étudiants, chercheurs, journalistes, réalisateurs, photographes, associations, membres de la communauté eux-mêmes...), elle interroge toutefois sur les pratiques des acteurs impliqués dans cette mise en visibilité.

Les zabbâlîn forment une communauté majoritairement copte ${ }^{2}$ présente dans plusieurs grandes villes égyptiennes (par exemple à Alexandrie, voir Farag et al. 1997, Winegar 2012) pour ramasser, trier et valoriser une partie du gisement de déchets produit quotidiennement par la ville. On peut préciser que le qualificatif de communauté est parfois critiqué, certains auteurs lui préférant celui de corporation (Florin 2010). Aujourd'hui, la communauté vit notamment de la collecte des déchets, de leur recyclage et de l'élevage de porcs ${ }^{3}$ nourris par les restes organiques même si on note depuis les années 1980 une évolution de la structure économique et sociale interne à la communauté (Furniss et Desvaux 2018), activités auxquelles il ne faut pas oublier d'ajouter des chauffeurs, boulangers, épiciers etc. qui accompagnent l'activité principale de retraitement des déchets qui caractérise la communauté. La particularité de cette situation, qui voit une communauté prendre en charge une large partie des déchets d'une agglomération aussi peuplée, s'explique historiquement et renvoie aux vagues de migrations rurales qui se sont mises en place à compter du xIx $x^{\mathrm{e}}$ siècle en Égypte et vers Le Caire plus particulièrement. Sans chercher à reprendre de manière exhaustive la mise en place historique de ce mode de fonctionnement singulier, on peut mettre en avant

1 Quartiers où vivent et travaillent les zabbâlîn.

2 Chrétiens orthodoxes rattachés à l'église d'Alexandrie.

3 Dont les cheptels se sont progressivement reconstitués depuis leur abattage en 2009 (Florin 2010). 
les grandes étapes de la constitution de ce système ayant abouti à la place de la communauté aujourd'hui. Depuis les premières mentions dans les travaux des commissaires sanitaires et administrateurs britanniques dans le Lancet $^{4}$ (Special Commissioner 1883, 1884, 1909a, 1909b, Balfour 1919a, 1919b, 1919c), la communauté a fait l'objet de nombreux travaux. Ainsi, plusieurs thèses et mémoires de master ont porté plus ou moins directement sur les chiffonniers depuis les travaux précurseurs de Shérif alHakim, Günter Meyer ou Ragui Assaad, jusqu'à ceux plus récents de Jamie Furniss, Gaétan du Roy et Bénédicte Florin entre autres. La plupart de ces recherches portent essentiellement sur la communauté du Muqattam dans le quartier de Manshiyyat Nâsir. La communauté occupe une place importante dans le folklore égyptien comme en témoigne le cinéma national. Des documentaires ont été réalisés (Marina of the Zabbaleen d'Engi Wassef en 2008, Garbage Dreams de Mai Iskander en $2009 . .$.$) , d'innombrables articles de presse abordent l'un ou l'autre aspect de$ la question et des romans ont été écrits sur la communauté comme Ahlam et les éboueurs du Caire de Fawziya Assaad (Assaad 2004), récit d'un paysan chrétien immigré à la capitale trouvant dans le commerce des déchets et l'élevage de porc une porte de sortie à sa condition désœuvrée. La réalisation de la fresque de l'artiste franco-tunisien El Seed (voir de Broqueville dans ce numéro) en 2016 a remis en visibilité le quartier par l'entrée artistique, dans la lignée de projets précédents - on songe aux lampadaires solaires installés en 2011 à Manshiyyat Nâsir en collaboration avec la coopération technique française et qui ont aujourd'hui cessé de fonctionner.

Cette introduction ${ }^{5}$ se donne pour objet de brosser les grands traits des ressorts de la surétude en général et appliquée au cas cairote, afin de poser des termes de réflexion pour le lecteur. Je ne ferai ici que survoler une analyse qui mériterait un travail bien plus poussé. II ne s'agit donc pas tant de mettre en avant les résultats d'une recherche menée sur le sujet que de proposer des pistes de réflexion, destinées à fournir des éléments de débats pour de futurs développements. Je m'attarderai ici dans un premier temps à effectuer un tour d'horizon de la littérature scientifique consacrée à la surétude avant de me pencher dans un second temps sur ce que ces éléments nous permettent de dire sur le cas cairote en particulier.

4 The Lancet est un journal médical anglais fondé en 1823 publiant de nombreuses contributions de scientifiques mais également d'administrateurs coloniaux tout en entretenant un réseau de journalistes scientifiques dans l'Empire Britannique.

5 Je tiens ici à remercier Jamie Furniss et Gaétan du Roy pour leurs relectures et leurs conseils. 


\section{ÉTUDIER LA SURÉTUDE}

Avant de se pencher sur les ressorts propres à l'analyse du cas concret qui nous intéresse ici, il est intéressant de se pencher sur un ensemble de travaux, certes restreint, qui s'est intéressé à la question de la "surétude ». Si les zabbâlîn représentent un exemple type de ce phénomène, comme nous I'appréhenderons dans la suite de cette introduction, ils ne sont pas les seuls à être concernés.

L'étude des enjeux liés à l'investissement répétés de certains terrains d'enquête entraînant les phénomènes de "sur-étude », " sur-enquête » ou " sur-recherche ${ }^{6}$ » sont très peu abordés par les chercheurs. On peut également noter que ces débats concernent principalement des travauxanglophones, et I'on ne retrouve que très peu de recherches francophones qui se penchent spécifiquement sur cette question (à l'exception notable de Chabrol 2008; le problème est parfois mentionné, voir par exemple Mahoudeau 2017). L'organisation d'une double session dédiée à ce sujet à la conférence annuelle de la Royal Geographical Society (RGS) en août 2018 (Button et Aiken 2018) ainsi que d'une journée "Jeunes chercheurs » au $\operatorname{LADEC}^{7}$ témoigne de cet intérêt grandissant même si on remarque que "le problème des communautés sur-étudiées, ironiquement, est en soi un sujet sous-recherché dans les sciences sociales» (Sukarieh et Tannock 2013 : 494). On remarque par ailleurs que ces débats ne se limitent pas à une discipline scientifique donnée (malgré une représentation importante des sociologues et des anthropologues) et s'intéressent à des communautés données (Chabrol 2008, Clark 2008, Bull 2010, Clark 2010), des lieux comme les camps de réfugiés (Sukarieh et Tannock 2013, Pascucci 2017) ou encore des approches méthodologiques, qu'elles soient qualitatives (Titterton et Smart 2008) ou quantitatives (Chetty 1996). Dans le contexte égyptien, on pourrait mettre en avant d'autres terrains ou sujets très investis comme la Cité des morts, le quartier d'Imbaba (en lien avec la présence de mouvements islamistes), ou plus récemment les graffitis de la Révolution.

Ces analyses mettent en avant plusieurs enjeux associés à la surétude qu'il est possible de scinder en deux ensembles : d'une part les conditions et les structures favorisant l'émergence de ces situations, de l'autre leurs conséquences sur les objets d'étude et notamment sur les personnes étudiées elles-mêmes.

6 Les travaux anglophones emploient le terme « over-research » (« over-researched communities », « over-researched places », etc.), j'emploierai ici le terme « surétude ». 7 Voir le programme ici : http://ladec.cnrs.fr/wp-content/uploads/2018/05/ProgrammeJJC-Politiques-de-la-surenque\%CC\%82te1_06_2018.pdf 


\section{Les conditions d'émergence de la surétude}

Dans I'article On the problem of over-researched communities: The case of the Shatila Palestinian refugee camp in Lebanon (Sukarieh et Tannock 2013), les auteurs proposent une grille d'analyse des facteurs permettant l'émergence de la surétude. L'article met en avant la parole des habitants du camp de Chatila (Liban), notamment au regard de leur statut de "recherchés ». Si les auteurs estiment que les phénomènes de surétude peuvent émerger partout, ils font toutefois ressortir trois caractéristiques-clés des communautés qui favorisent la mise en place de ces " aimants » à chercheurs : un trait marginalisant (pauvreté, indigènes, minorités etc.); l'expérience d'une forme de crise plus ou moins récente ou d'une résistance active à leur condition de marginalisation et enfin une localisation accessible (géographiquement ou politiquement) aux chercheurs étrangers (Sukarieh et Tannock 2013). Il s'agit de noter que c'est par différentes combinaisons de ces facteurs que la surétude se met en place, chacun jouant un rôle important. Ces trois caractéristiques s'appliquent directement au cas qui nous intéresse ici, car les zabbâlîn remplissent toutes les " conditions d'éligibilité » et confirment le modèle proposé : il s'agit d'une communauté stigmatisée sur plusieurs plans (minorité religieuse, stigmate associé au travail du déchet, élevage des porcs dans un pays à majorité musulmane) ayant fait l'expérience de plusieurs crises (modernisation des années 1980, délégation des années 2000, abattage des porcs en 2009) et offrant un terrain accessible à des personnes extérieures (proximité des zarrayeb du centre-ville cairote où résident les occidentaux, accueil le plus souvent favorable de la part des habitants habitués à la présence de membres extérieurs à la communauté dans les zarrayeb, relative stabilité politique de l'Égypte bien que ce dernier point doive être nuancé depuis la révolution de janvier 2011).

Ces différents éléments tendent à confirmer les hypothèses faites par les auteurs nommés ci-dessus et renvoient tous au même constat : c'est bien la place de l'enquêteur qui est ici centrale. Le choix de communautés stigmatisées et soumises à des aléas renvoie en effet aux choix méthodologiques, éditoriaux, médiatiques ou stratégiques effectués en amont. Considérer différents aspects (stigmatisation, crise, accessibilité) dont c'est l'agencement qui entraîne la surétude permet de considérer ce processus comme complexe et protéiforme. Dans ce cadre, ces situations doivent être comprises comme non-linéaires, et même si des principes similaires peuvent être identifiés, il est important de comprendre que toute situation de surétude ne relève pas toujours des mêmes facteurs, ou du moins à des degrés variables. Il est ainsi parlant de voir que dans notre exemple, chacun de ces facteurs peut se décliner de différentes manières, ce qui fournit un premier élément de compréhension à la persistance de la surétude des zabbâlîn. On retrouve en effet une stigmatisation multi-facettes et une série de crises qui ont nourri l'actualité autour de la communauté. Le dernier point, à savoir la facilité d'accès au terrain est également valable sur plusieurs plans : tout d'abord, comme le notent d'autres auteurs, la présence de longue date de réseaux internationaux dans la communauté suite à 
I'attention dirigée vers elle par l'action de Sœur Emmanuelle a entraîné la constitution d'infrastructures sociales (Desvaux 2017) qui facilitent grandement le travail d'investigation. De nombreux membres de la communauté parlent ainsi français et/ou anglais grâce aux réseaux de scouts ou aux cours donnés dans le quartier. De plus, les projets des ONG, le monastère de Manshiyyat Nâsir attirent de nombreux touristes et fidèles qui banalisent la présence de membres extérieurs et rendent plus aisé l'accès aux zarrayeb. L'effet cumulatif de ces infrastructures facilite le travail de terrain et renforce le phénomène de surétude, d'où une dimension tautologique : plus le quartier est investi par des étrangers et plus il est simple d'y travailler pour des acteurs extérieurs.

Ce dernier point ne va cependant pas de soi, car l'un des effets les plus mis en avant des situations de surétude est ainsi la "fatigue » ressentie par les enquêtés vis-à-vis de la présence importante et répétée d'observateurs étrangers (Clark 2008, 2010, Sukarieh et Tannock 2013, Cleary et al. 2016, Damon et al. 2017). L'absence de cette fatigue comme sentiment majoritairement partagé au sein des zabbâlîn (voir Didero dans ce numéro) pose donc question, et renvoie à la réception de la part de la communauté des démarches scientifiques ou journalistiques.

\section{Conséquences de la surétude}

Malgré sa (relative) absence du terrain cairote, le phénomène de fatigue est mentionné par la quasi-totalité des auteurs précités qui renvoient tous à des remontées des terrains témoignant de difficultés à entrer en relation avec les personnes enquêtées. On peut à nouveau mettre en avant plusieurs facteurs qui sont invoqués comme favorisant la lassitude des enquêtés face aux sollicitations répétées : des contraintes pratiques (financières, organisationnelles, temporelles...) qui compliquent les rencontres, une absence d'amélioration de la situation des enquêtés perçue par rapport à l'engagement dans le processus de recherche et aux espoirs qui y ont été associés, et enfin une indifférence à la démarche scientifique. La répétition du nombre de processus de recherche engagés sur des espaces ou auprès de communautés de petite taille tend donc à voir se multiplier ces phénomènes de fatigue. Une nouvelle fois, il faut considérer ces différentes dimensions pour saisir ce qui les favorise ou non dans le cas cairote. Concernant la communauté zabbâlîn, les contraintes pratiques sont (relativement) limitées : Le Caire est une ville facilement accessible, peu coûteuse pour qui bénéficie d'une bourse de recherche ou d'un salaire européens, et jouit d'un statut de ville internationale et diplomatique de par son statut de capitale égyptienne. Les évènements politiques récents n'ont troublé cet accès que très relativement et l'actualité des «Printemps arabes » a plutôt eu pour conséquence un afflux important de journalistes, chercheurs 
et documentalistes dans la foulée de la Révolution de $2011^{8}$. De plus, les plus grands, et les plus étudiés parmi les zarrayeb sont aisément accessibles : au-delà des infrastructures développées par une présence de longue date d'étrangers, les zarrayeb les plus étudiés sont également les plus accessibles : Manshiyyat Nâsir par micro-bus ou taxi, 'Izbit al-Nakhl est proche d'une station de métro.

Les deux éléments suivants sont intimement liés : I'intérêt porté au projet scientifique, ou de connaissance au sens large (qui permet d'inclure journalistes et documentaristes), est lié au retour que peuvent tirer les enquêtés de ce processus. Ils sont à mettre en lien avec des pratiques de recherches dites " extractives », qualifiées de coloniales par Linda Tuhiwai Smith (Smith 1999), qui renvoient à une conception classique du travail de terrain où le chercheur est placé en situation de collecte de données vis-à-vis de son objet d'étude. Dans ce cadre, les enquêtés ne tirent aucun bénéfice d'un processus de recherche où ils ne sont conviés que comme objets d'études, sans influence sur les termes de la recherche ou sur sa pratique. De plus, les enquêtés ont très rarement accès aux travaux finaux, souvent rédigés en langue étrangère et difficilement accessibles, et ne sont pas toujours satisfaits du résultat ${ }^{9}$. Cette mise à l'écart favorise une perception du travail de recherche comme inutile, car il n'apporte aucun élément et ne repose que sur le bon vouloir des personnes enquêtées qui donnent de leur temps et des savoirs locaux vernaculaires sans contrepartie. Les approches de recherche participative, via des groupes de recherche par exemple, qui ont été développées afin de tenter de dépasser les limites évoquées ci-dessus, peuvent toutefois générer ces phénomènes de fatigue au regard de I'implication demandée aux participants (Titterton et Smart 2008).

8 L'assassinat d'un doctorant italien en 2016 reflète le durcissement du régime depuis l'élection d'Abdel Fattah al-Sissi mais les travaux autour de la communauté jouissent encore (jusqu'à quand ?) d'une image "sympathique » et ne renvoient pas à des sujets politiquement sensibles au premier abord.

9 Le cas de Fawziya Assad est intéressant puisque la traduction arabe de son livre a provoqué le mécontentement de la famille dont elle racontait la vie. Autre exemple, le zabbâl dont le journaliste Peter Hessler a réalisé un portrait pour le New Yorker en 2014 (nous reviendrons plus longuement sur l'article plus bas) a rencontré des problèmes sur son lieu de travail (le quartier cossu de Zamalek où de nombreuses personnes lisent anglais) et a été accusé par ses clients d'espionnage et victime d'une campagne sur les réseaux sociaux qui demandait son éviction du quartier.

Voir sur les conséquences de l'article de Peter Hessler pour le zabbâl en question : Thomas S., What does the garbageman actually know?, Mish Kida, article paru le 9 octobre 2014.

URL : http://mishkida.tumblr.com/post/99622498835/what-does-the-garbagemanactually-know (consulté le 10 octobre 2018). 
On peut ici faire plusieurs remarques sur le cas des zabbâlîn, qui ne s'y limitent pas forcément. Si la présence d'observateurs extérieurs à la communauté peut parfois agacer, de nombreuses personnes tirent un bénéfice certain de cette ouverture sur l'extérieur (voir les contributions de Géry de Broqueville et de Gaétan du Roy dans ce numéro). On peut citer le cas du monastère du père Samaan (du Roy 2014) qui permet d'attirer des fonds via les réseaux chrétiens mais également des «fixeurs » (accompagnateurs) qui ont su s'installer comme guides en mettant en avant leur appartenance à la communauté en accompagnant notamment les journalistes souhaitant rédiger un article sur le sujet. Au-delà de ces exemples, les zabbâlîn ont su, particulièrement via l'influence des ONG, développer un discours « d'inversion du stigmate » Goffman 1963). En se réappropriant l'exceptionnalité projetée par d'autres sur eux, les membres de la communauté en sont ainsi venus à développer un discours valorisant à propos de leur activité, qui agit comme contre-discours aux clichés circulant sur les zabbâlîn dans la population égyptienne. Ces derniers se sont encore exprimés récemment lorsque le ministre de la justice Mahfouz Saber affirma en mai 2015 qu'il considérait que des fils de zabbâlîn n'avaient pas vocation à intégrer des fonctions prestigieuses, comme celle de juge. Si le ministre a dû démissionner à la suite d'une campagne sur les réseaux sociaux, cet épisode a permis de montrer la persistance des préjugés qui affectent toujours la société égyptienne et, partant, une communauté stigmatisée ${ }^{10}$ (voir également du Roy dans ce numéro). On retrouve ici, à travers le rôle des ONG, une dimension importante des situations de surétude mise en avant par la littérature sur le sujet, à savoir l'importance de l'existence d'une infrastructure constituée par les associations qui travaillent dans le quartier. Cette infrastructure est essentielle pour comprendre le fonctionnement de ces situations : elle permet d'une part de légitimer le travail des chercheurs et des journalistes en associant la médiatisation du cas à la possible captation de fonds de développement, elle permet également aux enquêteurs de bénéficier de " portes d'entrée » dans la communauté (Sukarieh et Tannock 2013, Pascucci 2017). Dans le cas cairote, I'influence des ONG a permis l'émergence d'un discours renversant la structure habituelle du stigmate : « on nous appelle les zabbâlin, mais ce n'est pas nous qui produisons des ordures, ce sont les habitants du Caire qui les produisent!» (cité dans Florin 2012).

La surétude de la communauté engendre donc des effets complexes, car si elle permet une plus grande facilité d'accès à des interlocuteurs locaux pour les enquêteurs, elle a également pour conséquence la constitution d'un discours préconstruit sur la communauté qui risque de masquer les aspérités spécifiques au terrain considéré, comme les jeux de pouvoir locaux par exemple

10 Voir Egypt's justice minister sacked over social class remarks, BBC News, article paru le 11 mai 2015. URL : https://www.bbc.com/news/world-middle-east-32688825 (consulté le 30/09/2018). 
(voir du Roy dans ce numéro pour une analyse poussée de cette dimension). La surétude produit donc des effets au sein de la communauté et peut faire I'objet d'enjeux et de tensions associés. Par exemple, les représentants auto-proclamés ou désignés ainsi de l'extérieur, canalisent la voix des zabbâlîn et la réduisent très largement à quelques personnes (souvent anglophones et/ou francophones) liées de près ou de loin aux réseaux développementalistes. Par ailleurs, la question du partage et de la redistribution des bénéfices liés à la captation de fonds de développement est un enjeu de taille, particulièrement dans une communauté fortement marquée par une hiérarchisation professionnelle renforcée par la structuration en familles plus ou moins influentes. Les ragots (fondés ou non) circulant autour de l'utilisation de l'argent récolté vont bon train, comme en témoigne le partenariat entre l'association Ruh el-Shabab (Spirit of Youth, fondée et dirigée par Ezzat Naem Gendy), la Ford Foundation et le cabinet Procter \& Gamble qui alimente les fantasmes. Si, comme on I'a vu, l'émergence de situations de surétude peut être analysée dans des contextes variés, il est important de considérer les ancrages locaux, nécessairement situés, de ces phénomènes pour en saisir les spécificités et les enjeux.

\section{LES ZABBÂLÎN : UN OBJET SURÉTUDIÉ ?}

\section{La construction historique de la surétude}

À ce stade de notre introduction, on comprendra que la question qui prélude à ce numéro, " les zabbâlîn : un objet sur-étudié ? " apparaît finalement rhétorique au regard de l'ambleur de la production de travaux académiques ou journalistiques. Dans le champ scientifique seulement ${ }^{11}$, et suite aux travaux précurseurs de Sherif El-Hakim et Kingsley Haynes (Haynes et El-Hakim 1979), de Günter Meyer (dont nous reproduisons un article paru en 1987 dans le journal Applied Geography), ou de Ragui Assaad (Assaad 1988, 1995, 1996), on compte, trois thèses francophones portant sur des thématiques liées à la communauté soutenues en 5 ans (Debout 2012a, du Roy 2014, Desvaux 2017). A cela s'ajoutent des travaux académiques francophones comme ceux de Bénédicte Florin (Florin 2010, 2011, 2012, 2015) et Safaa Monqid (Monqid 2011), sans compter les travaux anglophones de Fouda Abdel Motaal (Abdel Motaal 1997), Susanna Myllylä (Myllylä 2001), Elisa Wynne-Hughes (WynneHughes 2015), Mariz Tadros (Tadros 2017) ou encore Wael Fahmi et Kevin Sutton (Fahmi 2005, Fahmi et Sutton 2006, 2010), David Wilson (Jaligot et al. 2016), Maike Didero (Didero 2012) ou Jamie Furniss (Furniss 2012, Furniss et du Roy 2013, Furniss 2015, Furniss et Desvaux 2015, Furniss 2016, 2017) ou

11 Il serait impossible de recenser ici l'intégralité, ou même une partie significative, des articles de journaux consacrés à la communauté. 
la thèse en cours de Mohammed Rafi Arefin à l'Université du Wisconsin (Madison). Il faudrait ajouter à cette liste (non exhaustive) les travaux scientifiques arabophones dont je n'ai à ce jour pas connaissance et un nombre impressionnant de mémoire de Master qui constituent l'essentiel de la littérature scientifique sur le sujet (Boonen 1986, Volpi 1995, Dollet 2003, Ford 2003, Bosshard 2006, Butter 2006, Debout 2007, Piccardo 2007, Desvaux 2009, Milik 2010, Abadeer 2011, Mahieu 2011, Meijnen 2011, De Keyser 2012, Bakry 2015, Eisenschenk 2015, El Mahdy 2016, George 2016, Hussein 2017). De plus, les travaux de cabinets d'étude comme Environmental quality international (EQI) entre 1980 et 2000 (rapports recensés dans Furniss 2012, notamment via le travail de Mounir Neamatalla ou Berti Shaker, voir aussi du Roy dans ce numéro) ainsi que de CID Consulting, fondé par Leïla Iskandar qui a elle-même produit plusieurs travaux (Iskandar 1994, Iskandar et Tjell 2009), voire de l'Association pour la protection de l'environnement (APE) basée à Manshiyyat Nâsir (Walker 2005), ont fourni une somme non négligeable de connaissances sur la communauté. Les travaux de la Gesellschaft für Internationale Zusammenarbeit ${ }^{12}$ (GIZ, anciennement GTZ) ont participé aux nombreuses interventions dans le quartier de Manshiyyat Nâsir, bien que leur action (de 2010 à 2018) ne se soit pas limitée au zarrayeb. La plupart de ces recherches, destinées à la production de mémoire de Master, pourrait être problématique car ces travaux sont rarement associés à des engagements de long terme sur le terrain ou à des programmes plus larges et ne présentent donc pas, ou très peu, d'opportunité de retours. Cet aspect semble être contrebalancé par la sensibilisation de la communauté aux gains tirés de sa médiatisation renouvelée. Le bénéfice potentiel tiré par la communauté de sa médiatisation créée ainsi un effet "boule de neige ", en ce que le terrain investi par de précédents acteurs tend à être " préparé » pour les suivants en partie grâce à cette conscience de futurs gains possibles, sous les différentes formes qu'ils peuvent prendre, qui le rendent plus accessible. Au-delà de l'aspect financier du retour potentiel, la possibilité pour beaucoup de gens dans le quartier, en particulier parmi les plus jeunes, de pouvoir échanger avec des étrangers revêt en soi un intérêt. En plus du fait que la fréquentation d'étrangers est souvent valorisée, et à ce titre recherchée, ils en apprécient aussi la compagnie qui leur permet d'en apprendre plus sur les pays dont ils viennent et de se distraire un peu.

Si le premier élément de réponse à cet investissement auprès de la communauté peut être la facilité d'entrée du terrain mise en avant plus haut (infrastructure mise en place par les enquêtes et opérations de développement précédentes), il ne peut être considéré comme seul facteur de la surétude. II est important d'en mettre d'autres en avant qui expliquent cet intérêt que je souhaite décrypter ici à l'aune de deux aspects : le premier renvoie à

12 Agence de coopération internationale allemande. 
l'économie de production du savoir (académique et journalistique), le second à des considérations qui relèvent de l'imaginaire associé à une redéfinition des représentations associées à la communauté depuis l'extérieur.

\section{Quand le stigmate rejaillit sur le chercheur et/ou le journaliste : le prestige de l'enquête sur les zabbâlîn}

Le premier point que je souhaite développer ici ne renvoie que partiellement à la communauté en elle-même mais plutôt aux conditions extérieures de production de savoirs par les chercheurs et les journalistes. Il s'agit ici d'interroger ce qui pousse des chercheurs à se pencher sur les zabbâlîn en s'intéressant aux liens qui existent entre leur stigmatisation et le prestige associé à un travail académique ou journalistique. En effet, comme nous I'avons vu plus haut, la communauté centralise plusieurs formes de stigmatisation liée à sa religion (majoritairement copte), à son origine géographique (la Haute-Égypte) à son activité (le travail du déchet et l'élevage des porcs). Les ressorts des stigmates associés au travail du déchet sont par ailleurs mis en avant dans de nombreux travaux (Lhuilier 2005, Corteel et al. 2011) et ont été analysés dans le contexte cairote (Dollet 2003, Florin 2012 ou Florin dans ce volume). Le déchet et les activités associées comme "marqueurs négatifs » (Debout 2012b) touchent tant les membres de la communauté que leurs espaces de travail et de vie, un marqueur renforcé par la présence des élevages de porcs. La crise associée à la grippe H1N1 en 2009 a témoigné des craintes sanitaires associées à la présence des zarrayeb autour de la ville (Furniss 2012). Si les chercheurs et de nombreux journalistes étrangers comme égyptiens ont travaillé à dépasser ces clichés (on peut ici citer les articles de Dena Rashed pour Al-Ahram Weekly dans les années 2000), la présentation des conditions dans lesquelles s'effectue le recueil des données confronte physiquement l'enquêteur aux conditions de son terrain : saleté, odeurs, pollution des processus de recyclage sont alors autant de facteurs de dangerosité qui témoignent d'un engagement du corps même dans le recueil des données. Cette " assimilation » du stigmate du travailleur du déchet par l'enquêteur in-situ tend à renforcer le prestige ou la perception de la difficulté du travail de terrain depuis l'extérieur, d'où un gain d'autant plus grand pour ce dernier. La valorisation du travail est ainsi d'autant plus grande que le terrain a donné lieu à une mise en danger du corps (réelle ou perçue) de l'enquêteur qui en tire un bénéfice certain par la suite (Sontag 1966, Douglas 2001, Furniss 2008). Dans ce cadre, I'enquêteur n'a que peu d'intérêt à porter un regard critique sur une situation de marginalisation, pourtant critiquable en partie (voir du Roy dans ce numéro), ou à faire référence à un ensemble de travaux dont l'abondance relativise très fortement le mérite associé au travail de terrain.

Ce prestige associé aux enquêtes menées auprès des zabbâlîn est renforcé par la dimension multifactorielle des stigmates associés à la communauté. Le facteur religieux qui participe ainsi grandement de la médiatisation du cas, 
notamment dans le monde chrétien (minorité chrétienne persécutée dans un pays musulman), tend à construire la représentation d'une communauté placée dans une situation de danger qui est renforcée par les nombreuses crises qu'elle a pu connaître. Dans ce cadre, l'engagement du chercheur ou du journaliste, destiné à mettre à jour les formes de domination et de marginalisation subies par la communauté, peut être lu comme un acte moralement valorisant puisqu'il entre dans le cadre d'une défense de la " cause » zabbâlîn. On retrouve ici les mêmes mécanismes que ceux de la légitimation de la recherche sur les réfugiés palestiniens mis en avant plus haut (Sukarieh et Tannock 2013) où les enquêtés se voient associés à une lutte (dans le cas précédent la lutte palestinienne) qui valorise in fine l'engagement de l'enquêteur. Si on a pu voir que les zabbâlîn ont pu bénéficier de ce discours, il est important de noter que le gain agit dans les deux sens.

Il ne s'agit ici bien évidemment pas de montrer du doigt certains travaux ou $d^{\prime}$ 'en valoriser d'autres au regard d'une éthique quelconque mais bien de mettre en avant ce que je considère comme autant de facteurs permettant de comprendre comment la surétude de la communauté s'est construit. Le paradoxe entre, d'un côté, une relative facilité du travail de terrain mise en avant ci-dessus et, de l'autre, le prestige tiré d'un travail sur le sujet pose ainsi question au regard d'enquêtes éthiques qui verraient les conditions de leur déroulement rendues transparentes par leur auteur.

\section{Le romantisme postmoderne : entrepreneurialisme environnemental et néo-orientalisme}

Une autre piste d'interprétation de la surétude des zabbâlîn pourrait être à chercher du côté des champs sémantiques projetés sur, et réappropriés par, la communauté. On retrouve ainsi dans les discours véhiculés sur elle une forme de néo-orientalisme associée aux conditions de vie de la communauté qui se construit en lien avec un discours présentant les zabbâlîn comme une success-story de l'entrepreneurialisme environnemental.

La communauté fait dans un premier temps l'objet d'une fascination associée à la pauvreté et à la débrouille que je qualifie ici de "romantisme tératologique ». La tératologie est un terme qui fait référence à l'étude des « monstres » entendus soit comme un état de déviation d'un organisme à une norme donnée soit comme la survivance d'un état transitoire passé. Georges Canguilhem définit ainsi d'une part le monstre comme une «solution morphologique et fonctionnelle trouvée par la vie pour répondre à toutes les exigences du milieu »(Canguilhem 2013 : 120), et donc à des solutions trouvées à des situations particulières déviant d'une norme donnée. D'autre part, le monstre est prisonnier d'un passé par ailleurs dépassé, dans le cas des zabbâlîn, le renvoi aux chiffonniers parisiens du xIx ${ }^{\mathrm{e}}$ siècle : " la survivance d'une forme embryonnaire transitoire. Pour un organisme d'espèce donné, la monstruosité d'aujourd'hui [les zabbâlîn] c'est l'état normal d'avant-hier [les chiffonniers 
parisiens du xIx siècle] »(Canguilhem, 2003 : 230). La communauté zabbâlîn exerce une forme de fascination car elle occupe une place particulière dans le contexte cairote de par son activité : en évacuant et triant les déchets de la ville, elle représente une anomalie au regard des situations jugées normales dans les pays européens d'où proviennent une grande majorité des chercheurs et journalistes impliqués dans la surétude de la communauté. Si la présence de communautés de ramasseurs et recycleurs est courante dans les villes du Sud (catadores, cartoneros etc), elle apparaît comme la survivance d'une histoire passée pour les expériences urbaines du Nord, comme en témoigne le choix du terme de « chiffonnier » en français. Il renvoie à la survivance de pratiques jugées obsolètes par ailleurs, une attitude qui est également celle des pouvoirs publics quand on pense à la volonté régulièrement réaffirmée de moderniser la capitale.

La surétude des zabbâlîn est en partie liée à leur fourniture d'un service complet (depuis l'enlèvement au porte-à-porte à la valorisation) en l'absence de systèmes de gestion des déchets efficaces à l'échelle de l'agglomération de la part des gouvernorats cairotes. La communauté prend en charge une supposée prérogative de l'État au regard des modèles de développement occidentaux. L'originalité de cette forme de développement de services urbains (gestion des déchets), à laquelle s'ajoute la dimension valorisante du recyclage et de l'élevage des cochons, attire et ajoute à la spécificité du cas cairote. La success-story de la communauté, construite à travers les discours des ONG et de personnalités ayant lié leur carrière à elle (on pourrait ici citer Mounir Neamatalla, Marie Assaad ou Leïla Iskandar, voir du Roy 2014) valorise ainsi ce contre-discours dans le cadre d'une action civique, environnementale et entrepreneuriale. Le nom attribué par Leïla Iskandar au quartier, « Garbage village » ou « Garbage city » (Furniss 2012) reste aujourd'hui un terme employé par de nombreux zabbâlîn eux-mêmes pour mettre l'emphase sur la difficulté de leur situation, renforçant par là même la stigmatisation dont ils sont victimes mais qui permet de maintenir l'attractivité du quartier. Dans un contexte marqué par des inégalités mondiales croissantes et la montée des préoccupations environnementales, la communauté joue un rôle de catharsis en ce qu'elle représente une organisation originale, fondée sur la débrouillardise et obtenant de meilleurs taux de recyclage que $n^{\prime}$ importe quel pays du Nord, et fournissant des revenus à une population défavorisée. Dans ce système, la société de consommation capitaliste se voit doublement dédouanée de ses inconvénients : on y retrouve une réparation sociale, puisque nos déchets sont sources de richesses pour des populations stigmatisées - et donc de réduction d'inégalités, ainsi que d'un dédouanement de l'impact écologique de cette même consommation (puisque le recyclage est envisagé comme un moyen de limiter les externalités négatives des sociétés de consommation).

Toutefois, cette image de la communauté, largement construite de l'extérieur via les acteurs associatifs, journalistiques et académiques est à questionner. L'un des arguments régulièrement mis en avant pour démontrer l'importance de la communauté réside dans leur action environnementale : en 
débarrassant la ville de ses déchets, les zabbâlîn seraient ainsi en première ligne pour maintenir l'hygiène de la capitale et leurs taux de recyclage exceptionnels participeraient d'une action écologique. Si ces arguments se fondent sur une réalité observable, il est important de les nuancer. De précédents travaux (Debout 2012a) ont montré que les ramasseurs se voient dans I'incapacité physique (trop peu nombreux et équipés) de collecter les 15000 tonnes de déchets générées quotidiennement dans la ville. De plus, en se concentrant prioritairement sur les quartiers les plus aisés, la collecte sous la forme du service d'enlèvement n'est pas effectuée dans les quartiers populaires qui sont sillonnés par des récupérateurs ambulants (sariha ou nabbashin) membres ou non de la communauté. Au-delà des débats sur la portée écologique du recyclage (Grosse 2010), les conditions environnementales et sociales (conditions de travail des employés) dans lesquelles il est effectué tendent à limiter le poids de cet argument (fig. 1).

Un chiffre fréquemment évoqué pour mettre en avant la réussite de la communauté dans ce domaine pose également question. On entend souvent que les zabbâlîn recyclent $80 \%$ des déchets qu'ils collectent, soit un chiffre exceptionnel mis en rapport avec les taux de valorisation obtenus dans les pays dits « modernes » - par comparaison le taux de recyclage des déchets ménagers et

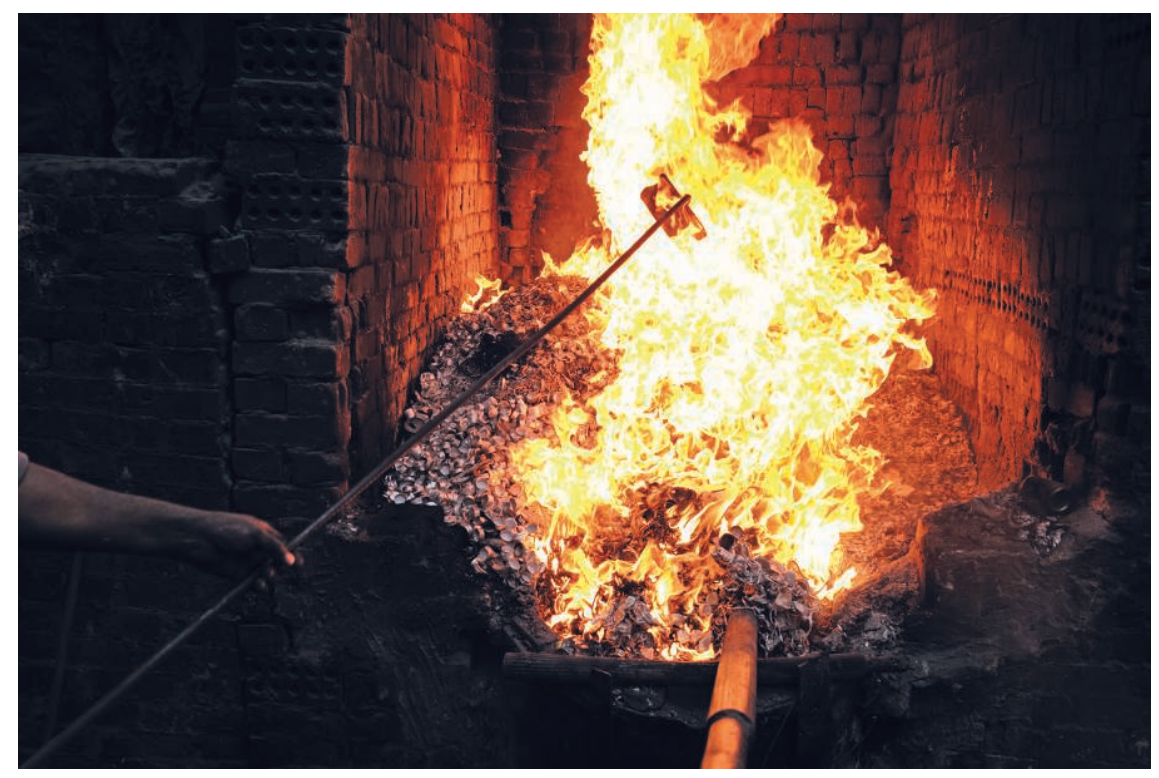

Figure 1 : Recyclage de I'aluminium à Manshiyyat Nâsir (C Degner)

Les objets en aluminium (canettes, barquettes alimentaires etc.) sont brûlés à l'air libre au sein du quartier. L'aluminium fondu reste au fond du creuset tandis que les impuretés flottent et sont retirées à l'aide d'une passoire. Cette pratique pose question au regard des impacts environnementaux sur les habitants du quartier (qui présente des densités de population parmi les plus élevées au monde) ou sur les travailleurs qui ne disposent d'aucune protection (le travail de l'aluminium est réputé comme dangereux au sein de la communauté). 
assimilés est, en France, estimé à 42\% par le Ministère de la transition écologique et solidaire. Si le fait qu'ils dépendent quasi-intégralement des revenus de la valorisation de la collecte tend à rendre crédibles des taux élevés de recyclage, ce chiffre qui est systématiquement mis en avant dans les travaux sur la communauté ne repose sur aucune étude statistique fiable et connue ${ }^{13}$. Le rôle valorisant associé aux zabbâlîn relève donc avant tout d'un discours qui n'est pas fondé sur des connaissances bien établies. De la même façon, il est extrêmement difficile de connaître la part précise des chiffonniers (sans compter que tous les sous-traitants des entreprises délégataires ne sont pas issus de la communauté) dans le système de gestion composite mis en avant dans les travaux de Lise Debout (Debout 2012b) compte tenu de la variété et de l'opacité des arrangements conclus à une échelle très fine (Furniss et Desvaux 2015).

La construction du discours valorisant autour du rôle de la communauté dans le milieu cairote renvoie en partie, à mon sens, à une forme de tératologie dans laquelle les zabbâlîn offrent une forme "monstrueuse » (en ce qu'elle dévie des modèles de gestion occidentaux) mais hautement originale, sorte de « fantasme post-moderne ». Dans cette acception, les formes de développement alternatives obtiendraient de meilleurs résultats que des services publics urbains modernes grâce à l'ingéniosité et à l'efficacité du « bricolage » appréhendés comme une forme de résistance et de maintien de pratiques « traditionnelles » face à un État égyptien failli et corrompu cherchant à imposer des solutions néolibérales déconnectées des réalités locales.

Il ne s'agit pas ici de prendre la défense de l'État égyptien mais plutôt de chercher à pointer les limites d'un tel discours sur les zabbâlîn qui ne repose pas sur des études fiables. Cette critique ne doit pas remettre en cause la validité des travaux menés par les chercheurs, les journalistes ou les associatifs mais être lue comme une mise en garde face à certains impensés des études menées au Caire. L'enjeu est de prêter attention à ne pas enfermer les formes prises par les expériences urbaines du Sud entre un romantisme valorisant la spécificité de la communauté (allant d'une forme de décroissance anticapitaliste à un entrepreneurialisme débridé) et une vision dystopique des futurs urbains qui fait écho à la construction des représentations orientalistes dénoncées par Anouar Abdel-Malek ou Edward Saïd (Abdel-Malek 1963, Said 2005). Cette tension est illustrée par la documentariste Julia Varga dans I'interview qui lui est consacrée dans ce numéro (voir aussi de Broqueville dans ce numéro). En prenant comme contrepoint de sa démarche ethnographique le documentaire Garbage Dreams, elle explique que « Le film proposait de très jolies

13 Il semblerait (je n'ai pas pu valider ces informations), suite à des discussions menées auprès de Jamie Furniss, que ce chiffre serait issu de l'un des rapports réalisés par EQI dans les années 1980. La méthodologie serait toutefois critiquable et de toute façon très datée à l'heure actuelle au vu des évolutions qu'a connues la communauté et son travail au cours des dernières décennies (Furniss et Desvaux 2018). 
images du quartier, mais c'était une sorte de conte de fées. Il y avait beaucoup de clichés, on restait dans les stéréotypes. Il y a des jeunes qui veulent s'en sortir mais ils en sont empêchés par la société et les discriminations et ils bravent tous les obstacles ». Le mérite de la communauté face aux obstacles mentionnés provoque ainsi une empathie ( "on soupçonne une hostilité autour d'eux et on se solidarise ») qui pousse à se méfier des biais cognitifs qui menacent tout travail sur la question, comme les débats autour de la parution de I'article de Peter Hessler en 2014 en ont témoigné ${ }^{14}$. Cet article, ayant connu un succès certain, prenait pour exemple le cas d'un zabbal (musulman) travaillant dans l'immeuble du journaliste situé dans le quartier cossu de Zamalek et cherchait à en dresser un portrait permettant de lire en creux la société égyptienne. Il a fait l'objet d'une critique adressée par Mohamed Rafi Arefin qui dénonçait l'orientalisme latent de l'auteur lorsqu'il mettait en avant, par exemple, le caractère exotique de la sexualité arabe. II lui reprochait notamment de ne pas avoir su situer son portrait dans une lecture plus générale de la place des déchets dans le paysage politique égyptien, lui préférant une approche «lubrique » de l'Égypte contemporaine, comme un écho aux descriptions orientalistes des siècles précédents ${ }^{15}$. Dans ce cadre, le monstre attire autant qu'il dégoute car « le monstrueux est du merveilleux à rebours, mais du merveilleux malgré tout» (Canguilhem, 2003 : 221).

\section{LA SURÉTUDE COMME PHÉNOMÈNE MULTIFORME}

Avant de conclure cette introduction, il paraît important de revenir sur les enjeux posés par la surétude. La question posée par le surinvestissement d'un terrain d'enquête pourrait être reformulée ainsi : surétudié voudrait-il dire qu'il n'y a plus rien à dire sur un sujet? Il ne faudrait en ce sens pas occulter les avantages liés à la présence importante de chercheurs, qui permet de multiplier les points de vue sur la question ainsi que de bénéficier des infrastructures posées historiquement par d'autres enquêteurs ou acteurs (Neal et al. 2016). Ces avantages sont toutefois largement occultés par le fait que les conditions concrètes de production de savoir (milieux académiques et journalistiques) tendent à valoriser des terrains originaux et les plus « purs " possibles, dans la lignée des fantasmes anthropologiques sur les communautés prémodernes

14 Voir l'article original : Hessler, Peter, «Tales of the Trash. A neighborhood garbageman explains modern Egypt», The New Yorker, 13 octobre 2014. Disponible en ligne (consulté le 27 septembre 2018) [https://www.newyorker.com/magazine/2014/10/13/ tales-trash].

15 Voir la critique complète: Mohammed Rafi Arefin, «The Dirty Details: A Response to "Tales of the Trash" », EdgeEffects, 30 octobre 2014. Disponible en ligne (consulté le 27 septembre 2018) [http://edgeeffects.net/tales-of-the-trash-response/]. 
(Sontag 1966). Dans ce cadre, c'est souvent l'individualisme et la « rétention » d'information qui sont privilégiés pour éviter de jeter le discrédit sur un terrain.

Cette remarque appelle d'autres questionnements, comme la question de savoir à partir de quand la surétude ne permet plus la multiplication des points de vue sur un sujet/thème donné. Les "zabbâlîn studies " nous confrontent donc à un paradoxe : à force d'être surétudiés, ils créent une déformation dans I'approche du Caire, issue de la projection de fantasmes divers sur cette communauté. Cet imaginaire associé aux chiffonniers du Caire n'est pas spontané et on peut retracer la constitution d'un mythe tant journalistique que scientifique. On y voit notamment comment les enjeux de regards « situés » (chrétiens, occidentaux etc.) prennent le pas sur la rigueur scientifique et alimentent des fantasmes plutôt que de les analyser sur un plan critique.

L'emploi du terme de déformation du regard n'est ici pas neutre, car il s'agit de faire référence à un phénomène de détournement du regard par la présence d'un objet « brillant » plus fort que les autres. Il est donc important de déconstruire les mythes entourant la communauté des zabbâlîn en reconsidérant les fondements scientifiques sur lesquels repose cette surreprésentation. Le risque est de laisser dans l'ombre d'autres pratiques ou groupes sociaux impliqués dans des activités proches de celles de la communauté. J'ai moi-même eu I'occasion de mettre en avant cette limite (Furniss et Desvaux 2018) qui tend à occulter, dans le champ des pratiques de récupération et de valorisation des déchets, des pratiques différentes, comme les filières des bekkia ${ }^{16}$ par exemple. L'exposition «Vie d'ordures » réalisée au MUCEM en 2015, au sein de laquelle I'exemple égyptien occupait une bonne place, a permis de mettre en lumière d'autres pratiques, espaces et acteurs impliqués dans la valorisation des déchets cairotes. Autre exemple : il semble que, d'un point de vue quantitatif, le recyclage opéré par les zabbâlîn doive être relativisé par rapport à l'ensemble des activités de recyclage présentes dans I'agglomération. Selon un rapport établi en 2008 (qui est la seule source disponible sur ce sujet à ma connaissance), les quartiers de zabbâlîn de Manshiyyat Nâsir et 'Izbit al-Nakhl ${ }^{17}$ réunis représenteraient ainsi « seulement » $53 \%$ du recyclage de plastique de l'agglomération (Plastic Technology Center et Industrial Modernization Center 2008), un chiffre qui, s'il peut sembler élevé, suggère que le rôle des zabbâlîn, traditionnellement surévalué dans les études sur la question, mériterait d'être défini plus précisément, d'une part, au sein des filières spécialisées de recyclage et, d'autre part, dans l'ensemble des activités de valorisation des déchets urbains. Selon

16 Les ramasseurs de bekkia sont des acheteurs ambulants qui font des " rondes » dans la ville sur des triporteurs (motorisés ou à pédales) afin de racheter des objets dont leurs propriétaires souhaitent se débarrasser.

17 Qui sont les plus gros zarrayeb et rassemblent la très grande majorité des capacités de recyclage de la communauté. II semble cohérent de penser que les chiffres de recyclage de ces quartiers représentent ceux de la communauté dans son ensemble. 
ce même rapport, les activités de recyclage d'Al Mishtal (Shubra el-Kheima) où ont été menés des entretiens autour du recyclage industriel représenteraient à eux seuls $23 \%$ du recyclage de l'agglomération, soit bien moins que Manshiyyat Nâsir (43 \%) mais plus qu'Izbit al-Nakhl (10\% selon Plastic Technology Center et Industrial Modernization Center 2008).

Les articles rassemblés dans ce numéro permettent de croiser plusieurs regards sur la communauté et sa mise en visibilité. Le texte de Gaétan du Roy est à lire comme un approfondissement des thématiques avancées dans cette introduction, et il permet de mettre en évidence le rôle particulier du quartier de Manshiyyat Nâsir dans l'imaginaire construit autour de la communauté. Pour l'auteur, ce lieu fait office d'hétérotopie où entrent en résonnance différents discours et rationalités qui confèrent au zarrayeb son statut si particulier dans la «mythologie » zabbâlîn. L'interview de Julia Varga permet, à travers son regard de documentariste et son expérience du Muqattam, de révéler certains des implicites qui préludent aux enquêtes non-académiques menées auprès des zabbâlîn. Elle y met en avant une lecture critique de la dimension orientaliste de ces sous-entendus, comme les images spectaculaires que renvoient les zarrayeb et l'aspect émotionnel associé à l'empathie générée par la situation difficile de la communauté sur lequel sont construits de nombreux travaux à son sujet. Elle en tire notamment comme conclusion la nécessité de porter un regard critique et conscient de ces clichés pour éviter de les reproduire et de les renforcer, afin de montrer la communauté dans sa complexité.

Les articles de Günter Meyer et Géry de Broqueville donnent une profondeur historique au travail sur et avec la communauté en combinant deux regards : celui du chercheur et de l'associatif. L'article de Günter Meyer, paru en 1987, pose les bases des futurs travaux sur les zabbâlîn, et permet de mettre en évidence les faibles progrès réalisés depuis cette date : on retrouve en effet mentionnées les origines et la structuration communautaires ainsi qu'une analyse des différents débouchés des matériaux de seconde main, notamment l'élevage des porcs, et la précarité des ramasseurs au regard de la lutte des autorités publiques contre la présence des charrettes dans le centre-ville. L'article de Géry de Broqueville, cofondateur d $\mathrm{ASMAE}^{18}$ en Belgique et en France, offre un témoignage précieux d'un engagement associatif de long terme, et laisse voir les paradoxes et complexités des soutiens aux zabbâlîn à travers I'action des ONG et de Sœur Emmanuelle en particulier. On voit comment les multiples interventions ont permis la médiatisation des chiffonniers

18 ASMAE Belgique est une association fondée en 1980 par Sœur Emmanuelle destinée à apporter une aide aux enfants démunis dans le monde. ASMAE France a été créée en 1985 par ASMAE Belgique mais les deux associations sont depuis 1990 complètement indépendantes du fait de désaccords autour de l'action de Sœur Emmanuelle (du Roy 2014). 
au-delà des clichés misérabilistes, permettant de lever de fonds et d'attirer des bénévoles au Caire.

La contribution de Maike Didero et Sarah Defrawy aborde les évolutions contemporaines de la communauté face aux diverses crises qu'elle a eu à affronter (à partir de la délégation de la gestion des déchets cairotes en 2002) pour montrer comment chacune d'entre elles a induit des transformations structurelles en son sein. La conclusion de l'article laisse voir une issue optimiste aux enjeux posés par la surétude puisque sont mis en avant le fait que l'étude de la communauté, au croisement de nombreux enjeux affectant la vie quotidienne de la capitale égyptienne, peut encore fournir à la fois des enseignements mais également de nouvelles interrogations qui prolongent le projet scientifique. Enfin, l'article de Bénédicte Florin permet d'ouvrir le débat à d'autres terrains et à une nécessaire dimension comparative en mettant en rapport deux communautés cairotes aux trajectoires similaires, à savoir les tanneurs et les zabbâlîn. Si les similitudes entre les deux communautés et leurs espaces de vie et de travail sont bien visibles, la médiatisation très différente des deux cas met en avant le rôle d'une pluralité de facteurs comme conditions d'émergence de la surétude : si les tanneurs sont pris dans des logiques de stigmatisation et de marginalisation comme les chiffonniers, leur cas ne suscite pas le même engouement.

La surétude renvoie ainsi à un phénomène complexe et multi-facette : le cas cairote fait ressortir des éléments d'explication qui lui sont propres, et appellent à une mise en contexte spécifique à chaque cas de surétude, mais qui mériteraient d'être mis en rapport avec des cas similaires au-delà de l'Égypte. En ce sens, si l'exemple de la communauté zabbâlîn semble paradigmatique des processus de surétude, il fait écho aux ressorts identifiés par d'autres (trait marginalisant, expérience de la crise, localisation accessible) et invite à plus d'analyses croisées sur ce phénomène et sur ses conséquences. En ce sens, la voix des personnes directement concernées, celle des zabbâlîn eux-mêmes, manque cruellement dans ce numéro. Cette absence appelle d'une part à une ouverture de la production de savoirs aux acteurs concernés et d'autre part à un approfondissement des quelques jalons posés ici qui, nous l'espérons, permettront de prolonger le débat autour de la surétude (et appelle donc à des études supplémentaires et complémentaires !). L'enjeu posé renvoie finalement au dépassement de pratiques extractivistes ancrées dans un contexte néocolonial (Smith 1999) et au développement de formes de méthodologie de terrain repensées. 


\section{BIBLIOGRAPHIE}

Abadeer C., 2011, Informality NGOs, and Cairo's trash collectors. Economic and social welfare policy in the authoritarian egyptian state, Bachelor of Arts in Political Science, sous la direction de B. Ansell, University of Minnesota.

Abdel-Malek A., 1963, " Orientalism in Crisis », Diogenes, Vol. 11, n 44, p. 103-140.

Abdel Motaal D., 1997, "The Zabbâlîn community of Muqattam. Reconstructing development: Women at the Muqattam settlement ", Cairo Papers in Social Sciences, Vol. 19, $\mathrm{n}^{\circ} 4$, p. 59-110.

Assaad F., 2004, Ahlam et les éboueurs du Caire, Grolley, Éditions de l'Hèbe.

Assaad R., 1988, "L'informel structuré. Les zabbâlîn du Caire », Peuples méditérranéens, Vol. 41-42, p. 181-192.

Assaad R., 1995, "La transformación del sistema de recolección de basura en El Cairo », Revista Mexicana de Sociología, Vol. 57, no 1, p. 167-182.

Assaad, R., 1996, "Formalizing the informal? The transformation of Cairo's refuse collection system », Journal of Planning Education and Research, Vol. 16, no 2, p. 115-126.

Bakry H., 2015, Grappling with Cairo's garbage: Informal sector integration as a means to urban sustainability, Masters of Science in Sustainable Development, sous la direction de K. Abdelhalim et H. Sewilam, American University in Cairo.

Balfour A., 1919a, "The problem of hygiene in Egypt. Lecture I: The causes of the problem », The Lancet, Vol. 5010, p. 417-421.

Balfour A., 1919b, "The problem of hygiene in Egypt. Lecture II: The solution of the problem - past and present », The Lancet, Vol. 5011, p. 465-470.

Balfour, A., 1919c, «The problem of hygiene in Egypt. Lecture III: The solution of the problem - present and future», The Lancet, Vol. 5012, p. 507-512.

Barthel P-A., 2017, « Le Caire : entre rentes et révoltes », in D. Lorrain (dir.), Métropoles en Méditerranée. Gouverner par les rentes, Paris, Presses de SciencesPo, p. 109-150.

Boonen A., 1986, Etude d'observation sur les différents types d'activités éducatives offertes par un centre à la population d'un village et d'un bidonville dans la banlieue nord-est du Caire, Mémoire de Master (non publié), Université Catholique de Louvain.

Bosshard C., 2006, Kamp und Müll. Eine Feldstudie über das Sheitern der Formalisierung eines informellen Wirtschaftszweiges in Ägypten, Mémoire de Master (non publié), Université de Zurich.

Bull J., 2010, « Research with Aboriginal Peoples: Authentic Relationships as a Precursor to Ethical Research », Journal of Empirical Research on Human Research Ethics, Vol. 5, $n^{\circ} 4$, p. 13-22.

Butter I., 2006, An Informal Waste Management System in Cairo: The garbage collectors and their settlements sous la direction de P. Nas, Leiden, University of Leiden.

Button C. et Aiken G., 2018, "Over-Researched Places », in RGS-IBG, Cardiff,

Canguilhem G., 2003 [1952], La connaissance de la vie, Paris, Vrin.

Canguilhem G., 2013 [1966], Le normal et le pathologique, Paris, Presses Universitaires de France. 
Chabrol F., 2008, « Enquêter en milieu convoité. Les terrains surinvestis de l'anthropologie », in D. Fassin et A. Benga (dir.), Les politiques de l'enquête. Épreuves ethnographiques, Paris, La Découverte, p. 229-244.

Chetty S., 1996, "The case study method for research in small-and medium-sized firms ", International Small Business Journal, Vol. 15, p. 73-85.

Clark T., 2008, "'We're over-researched here!': Exploring accounts of research fatigue within qualitative research engagements », Sociology, Vol. 42, p. 953-970.

Clark T., 2010, "On 'being researched': Why do people engage with qualitative research? ", Qualitative Research, Vol. 10, p. 399-419.

Cleary M., Siegfried N., Escott P., et al., 2016, « Super Research or Super-Researched?: When Enough is Enough... », Issues in Mental Health Nursing, Vol. 37, n 5, p. 380-382.

Corteel D., Le Lay S. (dir.), 2011, Les travailleurs des déchets, Toulouse, Érès.

Damon W., Callon C., Wiebe L., et al., 2017, « Community-based participatory research in a heavily researched inner city neighbourhood: Perspectives of people who use drugs on their experiences as peer researchers", Social Science \& Medicine, Vol. 176, p. 85-92.

De Keyser E., 2012, De zabbaleen: een evolutie naar formaliteit of een informele gemeenschap binnen de Egyptische samenleving?, Mémoire de Master en Langues et Cultures Orientales, sous la direction de G. Schallenbergh, Université de Gand.

Debout L., 2007, La libéralisation/modernisation en égypte, la possibilité d'une réforme de la gestion urbaine?, Mémoire de Master 2 en Aménagement de l'espace et Urbanisme, sous la direction de C. Lefèvre, Université Paris-Est Marne-la-Vallée.

Debout L., 2012a, Gouvernements urbains en régime autoritaire. Le cas de la gestion des déchets ménagers en Égypte, Thèse de doctorat en Géographie, Aménagement et Urbanisme, sous la direction de F. Scherrer et E. Verdeil, Université Lumière Lyon II.

Debout L., 2012b, " "Réseau mou" et intégration urbaine. Particularités du service de gestion des déchets ménagers ", Flux, Vol. 87, n 1, p. 7-17.

Desvaux P., 2009, Les impacts de la délégation de la gestion des déchets au Caire sur la filière informelle des ordures, Mémoire de Master 1 en Géographie, sous la direction de B. Florin, Université François Rabelais.

Desvaux P., 2017, L'arraisonnement des milieux urbains. Analyse des flux cataboliques au Caire (Égypte) et à Lyon (France), Thèse de doctorat en Géographie, sous la direction de M. Houssay-Holzschuch et E. Verdeil, Université Grenoble Alpes.

Didero M., 2012, "Cairo's informal waste collectors: A multi-scale and conflict sensitive perspective on sustainable livelihoods ", Erdkunde, Vol. 66, n 1 , p. 27-44.

Dollet S., 2003, Une communauté traditionnelle face à la modernité. Le cas des zabbâlîn du Caire, Mémoire de DEA en Science Politique Comparative, sous la direction d'O. Dabene, Université Aix-Marseille III.

Douglas M., 2001 [1967], De la souillure, essai sur les notions de pollution et de tabou, Paris, La Découverte.

du Roy, G., 2014, Le prêtre des chiffonniers ou la construction d'une autorité religieuse au Caire, entre charisme, tradition et clientélisme (1974-2014), Thèse de doctorat en Histoire, sous la direction de P. Servais et J. den Heijer, Université Catholique de Louvain.

Eisenschenk N., 2015, Exploring issues of relative deprivation in the Zabbaleen community in Cairo, Master thesis in Conflict Analysis and Resolution, sous la direction de 
M. Wohlfeld, University of Malta and the George Mason University's School for Conflict Analysis and Resolution (USA).

El Mahdy H., 2016, The reform of solid waste management in Cairo : Lessons learned from international models, Master of Arts thesis, sous la direction de G. Barsoum, American University in Cairo.

Fahmi W., 2005, «The impact of privatization of solid waste management on the Zabbaleen garbage collectors of Cairo », Environment and Urbanization, Vol. 17, n 2, p. 155-170.

Fahmi W. et Sutton K., 2006, "Cairo's Zabbaleen garbage recyclers: Multinationals' takeover and state relocation plans», Habitat International, Vol. 30, p. 809-837.

Fahmi W. et Sutton K., 2010, "Cairo's Contested Garbage: Sustainable Solid Waste Management and the Zabbaleen's Right to the City », Sustainability, Vol. 2, p. 1765-1783.

Farag M., Ramadan M., El-Sebaie O., et al., 1997, "Field study on plastic recycling in Alexandria city: Case study in Montazah district », Bulletin of High Institute of Public Health, Vol. 27, n 3, p. 461-476.

Florin B., 2010, "Réforme de la gestion des déchets et reconfigurations des territoires professionnels des chiffonniers du Caire », Géocarrefour, Vol. 85, n² 2, p. 109-118.

Florin B., 2011, "Dimensions spatiales d'une crise : les stratégies des zabbalîn (chiffonniers) du Caire face à la réforme du système de gestion des déchets », in D. Corteel et S. Le Lay (dir.), Les travailleurs des déchets, Toulouse, Érès, p. 69-93.

Florin B., 2012, "Résister, s'adapter ou disparaître : la corporation des chiffonniers du Caire en question », in Y. Bonny et al. (dir.), Espaces de vie, espaces enjeux entre investissements ordinaires et mobilisations politiques, Rennes, Presses Universitaires de Rennes, p. 129-145.

Florin B., 2015, "Les chiffonniers du Caire. Soutiers de la ville ou businessmen des ordures? », Ethnologie française, Vol. 153, p. 487-498.

Ford K., 2003, Re-conceptualizing sustainability: The role of outsiders and the importance of human development, Mémoire de Master (non publié), University of Minnesota.

Furniss J., 2008, "Purity and pollution between researcher and researched: Barriers to ethnography among a community of Egyptian garbage collectors », communication donnée le 26 avril 2008, Oxford Researching Africa Day, Oxford.

Furniss J., 2012, Metaphors of waste: Several ways of seeing "development" and Cairo's garbage collectors, $\mathrm{PhD}$ thesis in Philosophy, sous la direction de N. Gooptu, University of Oxford.

Furniss J., 2015, «Alternative framings of transnational waste flows: Reflections based on the Egypt - China PET plastic trade », Area, Vol. 47, n 1, p. 24-30.

Furniss J., 2016, "Postrevolutionary land encroachments in Cairo: Rhizomatic urban space making and the line of flight from illegality ", Singapore Journal of Tropical Geography, Vol. 37, no 3, p. 310-329.

Furniss J., 2017, "What type of problem is waste in Egypt? ", Social Anthropology, Vol. 25, no 3, p. 301-317.

Furniss J. et Desvaux P., 2015, " Hybridations formelles informelles. Gestion des déchets au Caire après la délégation à des entreprises internationales », in K. Bennafla (dir.), Acteurs et pouvoirs dans les villes du Maghreb et du Moyen-Orient, Paris, Karthala, p. 219-238. 
Furniss J. et Desvaux P., 2018, "L'État et la valorisation informelle des déchets au Caire : renforcement des logiques marchandes et prédation économique », in S. Jaglin et al. (dir.), Du rebut à la ressource. Valorisation des déchets dans les villes du Sud, Paris, Éditions AFD, p. 135-152.

Furniss J. et du Roy G., 2013, « Mission évangélisatrice et mission développementaliste : deux manières de filmer les chiffonniers du Caire », in E. Gangnat et al. (dir.), Mission et cinéma. Films missionnaires et missionnaires au cinéma, Paris, Karthala, p. 274-291.

George N., 2016, Second-chance programs addressing early school leavers: A SWOC analysis of an intervention in a marginalized urban area in Cairo, Master thesis in Public Administration, sous la direction de G. Barsoum, American University in Cairo.

Goffman E., 1963, Stigma. Notes on the management of spoiled identity, Englewood Cliffs, Prentice-Hall.

Grosse F., 2010, "Is recycling "part of the solution"? The role of recycling in an expanding society and a world of finite resources », SAPIENS, Vol. 3, p. 1-17.

Haynes K. et El-Hakim S., 1979, «Appropriate technology and public policy: The urban waste management system in Cairo », Geographical review, Vol. 69, n 1, p. 101-108.

Hussein M, 2017, Middle class imaginaries of Cairo's waste: The Zabbaleen's story retold, Master of Arts thesis in Gender and Women's Studies in the MENA, sous la direction de M. Rieker, American University in Cairo.

Iskandar L., 1994, Mokattam garbage village. Cairo, Egypt, Cairo, Stallion Graphics.

Iskandar L., Tjell J., 2009, «Editorial. Cairo: A colossal case of waste mismanagement to learn from », Waste Management and Research, Vol. 27, n 10, p. 939-940.

Jaligot R., Wilson D., Cheeseman C., et al., 2016, «Applying value chain analysis to informal sector recycling: A case study of the Zabbaleen », Resources, Conservation and Recycling, Vol. 114, no p. 80-91.

Lhuilier D., 2005, "Le "sale boulot" », Travailler, Vol. 14, n 2, p. 73-98.

Mahieu M., 2011, L'éducation non-formelle à l'égard des enfants travailleurs chez les chiffonniers du Caire. Une approche anthropologique du déchet, Mémoire de Master (non publié), Université Libre de Bruxelles.

Mahoudeau A., 2017, "Une colère électrique. Les camps de réfugiés palestiniens à Beyrouth », Savoir/Agir, Vol. 42, no 4, p. 111-118.

Meijnen R., 2011, Planning for machine and marriage. Agency of young male garbage workers in Cairo, in interaction with developmental and social structures, Mémoire de Master (non publié), Université de Leiden.

Milik S., 2010, Assessment of solid waste management in Egypt during the last decade in light of the partnership between the Egyptian government and the private sector, Master Of Arts thesis in Political Science, sous la direction de I. Elnur, American University in Cairo. Monqid S., 2011, "La gestion des déchets ménagers au Caire : les habitants en question », Égypte/Monde arabe, Vol. Ille Série, no 8, p. 85-105.

Myllylä S., 2001, "NGOs in urban environmental governance: Waste recycling in Cairo », in A. Tostensen et al. (dir.), Associational life in African cities: Popular responses to the urban crisis, Stockholm, Nordiska Afrikainstitutet, p. 198-217. 
Neal S., Mohan G., Cochrane A., et al., 2016, "'You can't move in Hackney without bumping into an anthropologist': why certain places attract research attention ", Qualitative Research, Vol. 16, n 5, p. 491-507.

Pascucci E., 2017, «The humanitarian infrastructure and the question of over-research: reflections on fieldwork in the refugee crises in the Middle East and North Africa », Area, Vol. 49, p. 249-255.

Piccardo L., 2007, Cooperative and integrational projects in Cairo's urban areas: The case of the Zabbaleen of Moqattam, Mémoire de Master (non publié), Universita Cattolica Del Sacro Cuore (Milano).

Plastic Technology Center \& Industrial Modernization Center, 2008, National study: To reach a development strategy \& action plan on how to upgrade the Egyptian Plastic Recycling Sector to face its future challenges and attract Foreign Direct Investment. Plastic recycling sector. Final report, Cairo, Ministry of Trade and Industry: Technological Centers Sector.

Said E., 2005 [1978], L'orientalisme : I'Orient créé par l'Occident, Paris, Éditions du Seuil.

Smith L., 1999, Decolonizing methodologies: Research and indigenous peoples, London, Zed Books.

Sontag S., 1966, Against interpretation and others essays, Cambridge, M.I.T. Press.

Special Commissioner, 1883, "Cholera in Egypt », The Lancet, n¹22, 482-483 p.

Special Commissioner, 1884, "The new sanitary direction in Egypt », The Lancet, $\mathrm{n}^{\circ} 123$, $593 \mathrm{p.}$

Special Commissioner, 1909a, "The cleansing of Cairo », The Lancet, Vol. 173, n 4476, p. $1720-1723$.

Special Commissioner, 1909b, "The cleansing of Cairo (2) », The Lancet, Vol. 173, n 4477, p. 1782-1784.

Sukarieh M., Tannock S., 2013, « On the problem of over-researched communities: The case of the Shatila Palestinian refugee camp in Lebanon », Sociology, Vol. 47, n 3, p. 494-508.

Tadros M., 2017, "The undesirables of Egypt. A story of persecution and defiance », in N. van Doorn-Harder (dir.), Copts in context: Negotiating identity, tradition, and modernity, Columbia, The University of South Carolina Press, p. 34-51.

Titterton M., Smart H., 2008, "Can participatory research be a route to empowerment? A case study of a disadvantaged Scottish community », Community Development Journal, Vol. 43, no 1, p. 52-64.

Volpi E., 1995, Development and community organization: Informal garbage collectors in Cairo, Mémoire de Master en Sociologie et Anthropologie, American University in Cairo.

Walker W., 2005, The Torah Zabbaleen. From tin shacks to high rises, Cairo, Association for the Protection of the Environment.

Winegar J., 2012, "Taking out the trash: Youth clean up Egypt after Mubarak », in J. Sowers, C.Toensing (dir.), The journey to Tahrir: Revolution, protest ans social change in Egypt, London, Verso, p. 64-72.

Wynne-Hughes E., 2015, " Governing through Garbage-City tourism », Interventions, Vol. 17, $n^{\circ} 6$, p. 839-852. 